\title{
Experimental analysis of the high-order harmonic components generation in few-layer graphene
}

\author{
Andreea I. Hadarig • Carlos Vázquez • Miguel Fernández • \\ Samuel Ver Hoeye · George R. Hotopan · René Camblor • \\ Fernando Las Heras
}

Received: 23 June 2014/Accepted: 13 August 2014/Published online: 20 September 2014

(C) Springer-Verlag Berlin Heidelberg 2014

\begin{abstract}
In this work, the nonlinear electromagnetic response of a few-layer graphene sheet is experimentally analyzed. The few-layer graphene sheet is obtained through mechanical exfoliation from highly ordered pyrolytic graphite and embedded in a rectangular waveguide structure which is used to guide the exciting and the output signals. The nonlinear electromagnetic response of the graphene sheet is exploited to implement a frequency multiplier in which the output signal, in the $330-500 \mathrm{GHz}$ frequency band, will be obtained as a high-order harmonic component of the input signal, in the $26-40 \mathrm{GHz}$ frequency band. Due to the particular selection of the input and output frequency ranges, the behavior of several harmonic components, from order 9 to 17 , can be characterized. The analysis will be focused on the frequency response of the graphene sheet, the influence of the input power on the output signal and the differences between the even- and odd-order harmonic components. Finally, it will be shown that the developed assembly can be used as $\mathrm{THz}$ signal source based on high-order frequency multiplication.
\end{abstract}

\section{Introduction}

Since it was isolated in 2004 [1], graphene has attracted the attention of a great number of researchers and scientific institutions all over the world, due to its unexpected behavior and properties. Its truly two-dimensional crystal structure and the linear energy spectrum of electrons and

A. I. Hadarig · C. Vázquez · M. Fernández ( $₫)$

S. Ver Hoeye · G. R. Hotopan - R. Camblor · F. Las Heras

Edificio Polivalente, Modulo 8, Planta 1, Campus Universitario

de Viesques, 33204 Gijón, Spain

e-mail: mfgarcia@tsc.uniovi.es holes give rise to linear effects which have been theoretically and experimentally demonstrated, such as the modification of the quantum Hall effect, the Klein tunneling or the minimal electrical conductivity, among many others [2].

On the other hand, a great number of nonlinear phenomena, including second [3-7]- and third [8-10]-harmonic generation and frequency mixing [11-13], have also been theoretically predicted and experimentally demonstrated. Moreover, the previously reported works have shown that the microscopic mechanisms that cause such effects in graphene are very different and strongly enhanced when compared with those observed in conventional semiconductors [2, 14]. High-efficiency second- and third-harmonic generation effects have been achieved in monolayer graphene-based transistors, from relatively low frequencies up to the millimeter-wave frequency band [1517], and they have also been experimentally observed in few-layer graphene sheets $[6,8,11,13]$. Therefore, such results can constitute the basis for the development of a radically new generation of electronic devices. However, the maximum achievable operation frequency is strongly limited by the parasitic elements associated with their relatively complex structure and the actual manufacturing processes. In this way, the theoretically predicted flat frequency response of graphene from low frequencies to the $\mathrm{THz}$ band [3] cannot be fully exploited.

In this work, the capability of few-layer graphene to generate by itself high-order harmonic components of an incident electromagnetic wave will be experimentally analyzed. The few-layer graphene is obtained through mechanical exfoliation from highly ordered pyrolytic graphite (HOPG). Next, is integrated together with a stripline structure [18] suitable to deal with the considered frequency bands. The $26-40 \mathrm{GHz}$ exciting signal is guided 

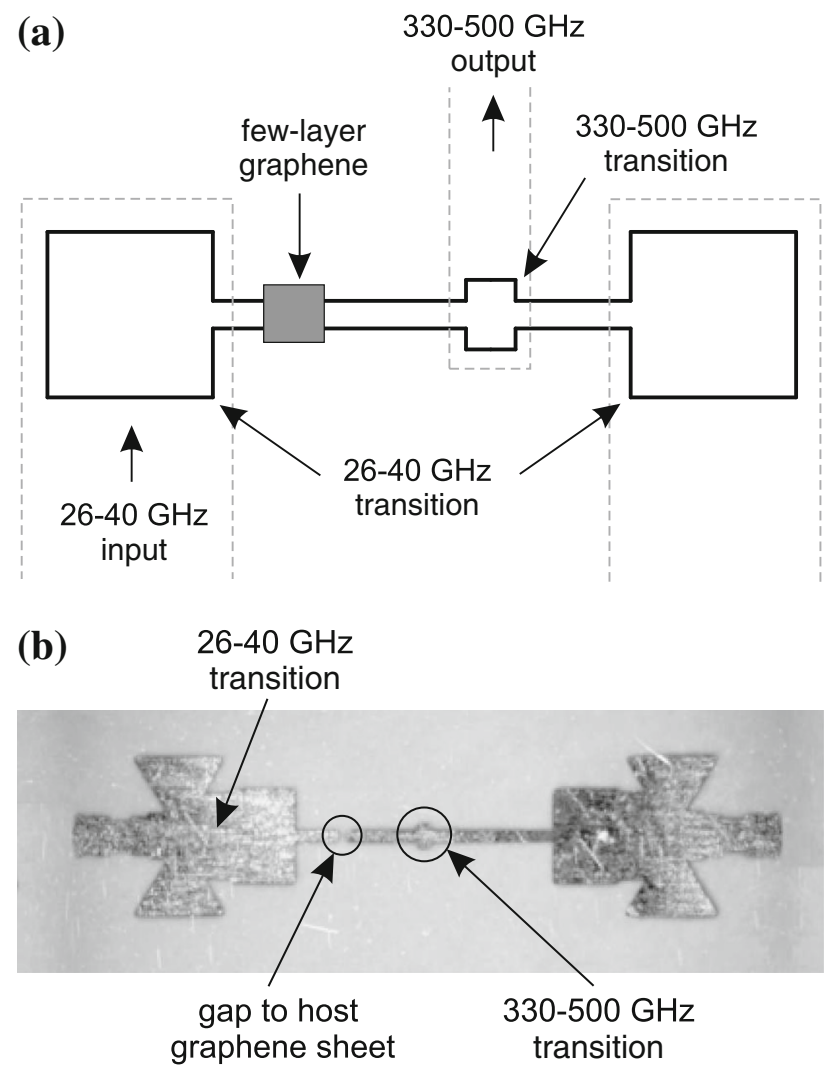

Fig. 1 a Schematic of the stripline circuit. b Picture of the implemented stripline circuit

to the few-layer graphene through a rectangular metallic waveguide. The output signal, generated due to the nonlinear behavior of the graphene, in the $330-500 \mathrm{GHz}$ frequency range, is extracted by using another rectangular metallic waveguide. Due to the selected input and output frequency ranges, the excitation of harmonic components from order 9 to 17 on the few-layer graphene structure will be analyzed, focusing on the output power, the frequency response and the differences between the even- and oddorder harmonic components. In addition, it will be shown that the developed assembly represents a proof of concept which demonstrates that graphene can be used in a single stage as $\mathrm{THz}$ signal source based on frequency multiplication.

\section{Experiment design}

\subsection{Stripline circuit with few-layer graphene}

The few-layer graphene sheet is obtained by mechanical exfoliation from a HOPG block. This sheet is structured through a laser-based process in order to implement a structure with the adequate size and shape to be integrated
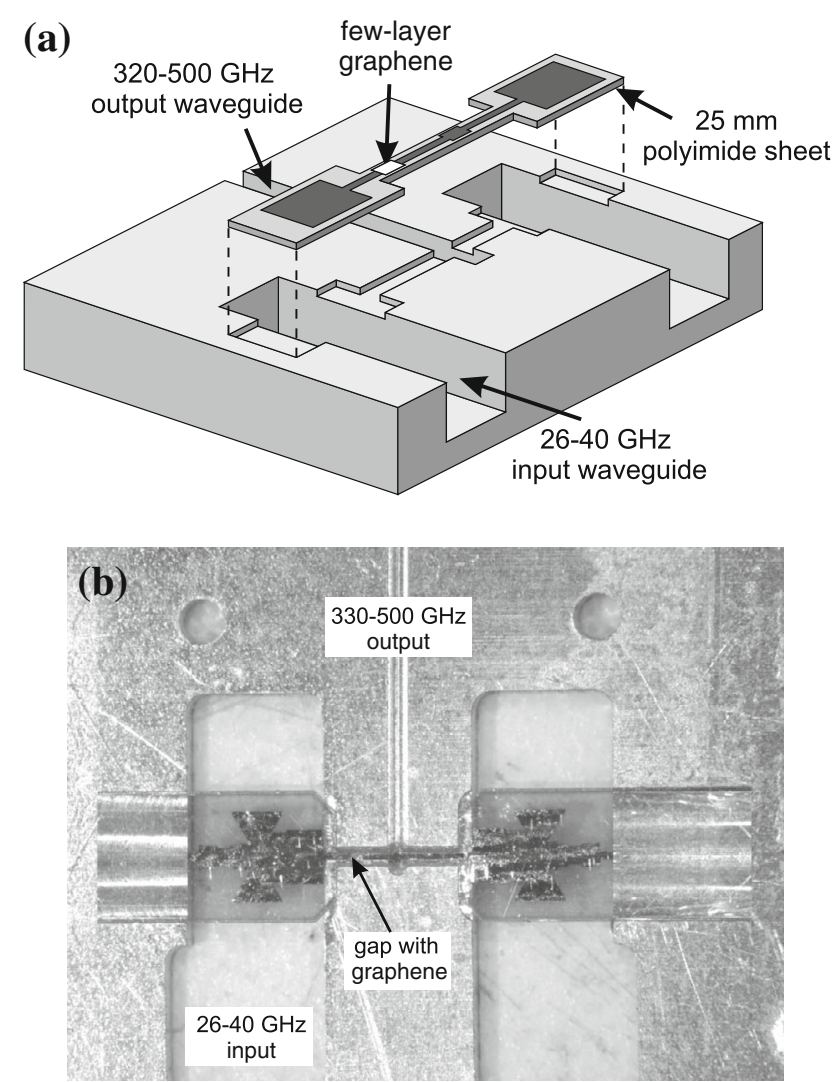

Fig. 2 a Schematic three-dimensional structure of the waveguide block and the stripline circuit. Note that only one of the two identical halves has been represented. b Picture of the stripline circuit with graphene placed in the waveguide block

together with the stripline circuit. As has been schematized in Fig. 1a, the stripline circuit is composed by one 26-40 GHz waveguide-to-planar circuit transition [18], and other one designed to work in the $330-500 \mathrm{GHz}$ frequency range. The transitions are connected by a short length transmission line which includes a gap to host the few-layer graphene sample. Another $26-40 \mathrm{GHz}$ waveguide-to-microstrip transition is added to achieve broad bandwidth operation.

The circuit metallization and the contacts with the graphene sample have been implemented by depositing gold on a $25 \mu \mathrm{m}$-thickness sheet of polyimide through an sputtering process. The stripline assembly is completed by adding a $25 \mu \mathrm{m}$-thickness polyimide sheet cover. Figure $1 \mathrm{~b}$ shows a picture of the implemented stripline circuit, without the top cover.

\subsection{Waveguide structure}

In order to excite the stripline circuit with few-layer graphene and to extract the generated high-order harmonic components, the circuit is embedded in a metallic 
waveguide structure. Such structure is composed by two $26-40 \mathrm{GHz}$ waveguide sections. One of them is used to propagate the input signal to the stripline circuit, while the other is used to achieve input impedance matching over the whole considered frequency range. The output signal is coupled to the $330-500 \mathrm{GHz}$ waveguide through the corresponding transition (see Fig. 1a). As represented in Fig. 2a, all the waveguide-to-microstrip transitions are inserted along the E-plane of the corresponding waveguide. Finally, the waveguide block includes a channel to place the stripline circuit. The height of the channel portion which is between the two $26-40 \mathrm{GHz}$ waveguides is $50 \mu \mathrm{m}$. It has been sized to avoid the propagation of waveguide modes around the stripline circuit. Figure $2 \mathrm{a}$ schematizes one half of the waveguide block and the stripline circuit without the top polyimide cover.

The input signal propagates along the waveguide under the fundamental $T E_{10}$ mode [18]. At the input transition, the waveguide $T E_{10}$ mode is converted into a TEM mode which will be propagated on the stripline circuit, exciting the graphene sample. Due to the nonlinear behavior of the graphene, several harmonic components of the input signal will be generated and propagated on the stripline circuit. The high-order harmonic components whose frequency is in the $330-500 \mathrm{GHz}$ range will be coupled to the output waveguide through the smallest transition represented in Fig. 1.

The assembly represented in Fig. 2a has been designed and optimized in order to achieve impedance matching and good power transfer from the waveguide to the stripline circuit along the $26-40 \mathrm{GHz}$ frequency range, to maximize the power coupled to the output $330-500 \mathrm{GHz}$ waveguide and to avoid the propagation of waveguide modes on the channel which interconnects the waveguides. The design and optimization processes have been performed with the aid of the finite element electromagnetic simulator Ansoft HFSS. The fundamental design parameters are the shape of the transitions, their position with respect to the back-shorts and the height of the channel between the waveguides. The relevant S parameters [18] of the whole assembly composed by the stripline circuit embedded in the waveguide structure have been represented in Fig. 3. Part a) shows the input impedance matching, evaluated at the input port indicated in Fig. 2, and the power transfer between the two 26-40 GHz waveguides. As can be observed, good impedance matching and low loss power transfer have been achieved over most of the 26-40 GHz frequency range. On the other hand, the electrical behavior of the high-frequency section has been analyzed by computing the $\mathrm{S}$ parameters of a substructure composed by the 330-500 GHz waveguide, the corresponding transition and the two segments of transmission line connected to it. The obtained results are shown in Fig. 3b. Note that the
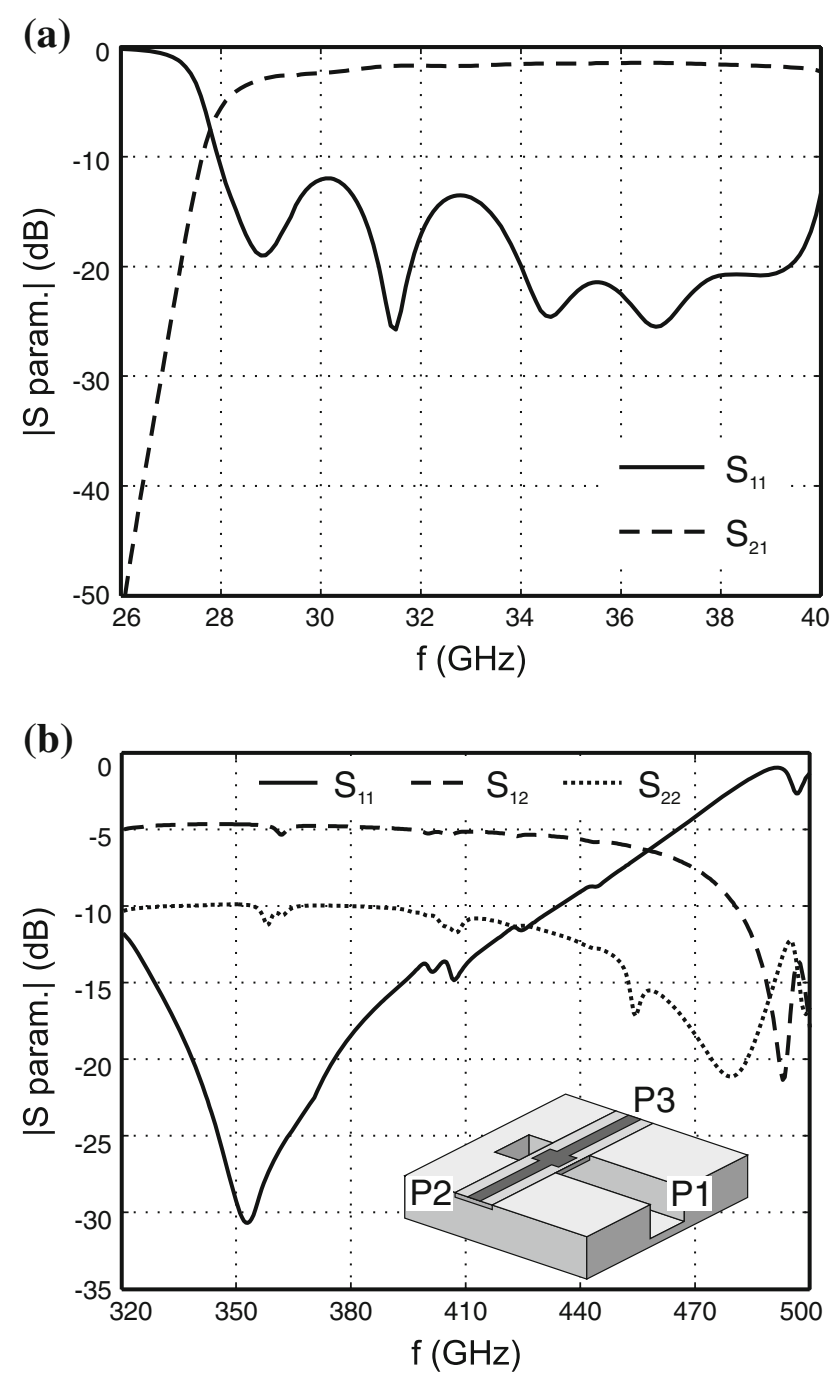

Fig. 3 Electrical behavior of the assembly composed by the stripline circuit embedded in the waveguide block. a Relevant $S$ parameters of the low-frequency section. b Relevant $\mathrm{S}$ parameters of the highfrequency section

optimum operation ranges are located between 27.5 and $40 \mathrm{GHz}$ at the input section and between 320 and $470 \mathrm{GHz}$ at the output, which will condition the bandwidth of the whole assembly.

\section{Experimental characterization}

\subsection{Measurement setup}

The measurement setup has been represented in Fig. 4. A signal source and a broad bandwidth amplifier are used to generate the $26-40 \mathrm{GHz}$ input signal. The amplified signal is connected to the input port of the waveguide block through a commercial coaxial to waveguide transition. The other $26-40 \mathrm{GHz}$ waveguide port is terminated with a 


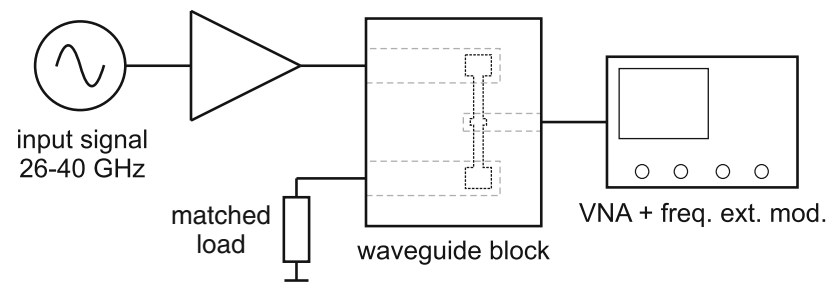

Fig. 4 Measurement setup

matched load. An Agilent PNA-X Vector Network Analyzer (VNA) equipped with a Virginia Diodes Inc. frequency range extender module is used to measure the power of the output signal in the $330-500 \mathrm{GHz}$ frequency range, generated through frequency multiplication due to the nonlinear electromagnetic behavior of graphene.

The frequency of the input and output signals is limited by the bandwidth in which the corresponding waveguides operate under fundamental $T E_{10}$ mode and by the bandwidth of the measurement equipment. Since the frequency of the input signal is in the $26-40 \mathrm{GHz}$ range, the harmonic-order values that provide an output signal in the $330-500 \mathrm{GHz}$ frequency range can vary from $N=9$ to $N=17$. Although the whole output frequency range is given by $[N \times 26-N \times 40] \mathrm{GHz}$, the output waveguide and the measurement system limit the output frequency range in which the signal power can be evaluated. Therefore, for most values of $N$, only a portion of the range between 26 and $40 \mathrm{GHz}$ can be considered.

\subsection{Odd-order harmonic components}

The evolution of the output power of the odd-order harmonic components, from $N=9$ to $N=17$, has been represented in Figs. 5 and 6 . The output power of the selected harmonic components has been evaluated considering different input signal power values, in $2 \mathrm{~dB}$ steps, with a $-6 \mathrm{dBm}$ maximum. The vertical lines indicate the limit of the nominal operation range, which is calculated for each value of $N$ taking into account the bandwidth of the input waveguide and the measurement system. Data obtained out of such nominal range have been represented with dashed line. Note that, although operation outside the indicated limits is possible, it is non-optimum and should not be considered.

As can be observed from Fig. 5, the maximum reached power in the case of the 9th, 11th and 15th harmonic components is around $-40 \mathrm{dBm}$. Although the theory predicts nearly flat frequency response from low frequencies to the $\mathrm{THz}$ band, relatively high ripple level is obtained. This is due to impedance mismatching between
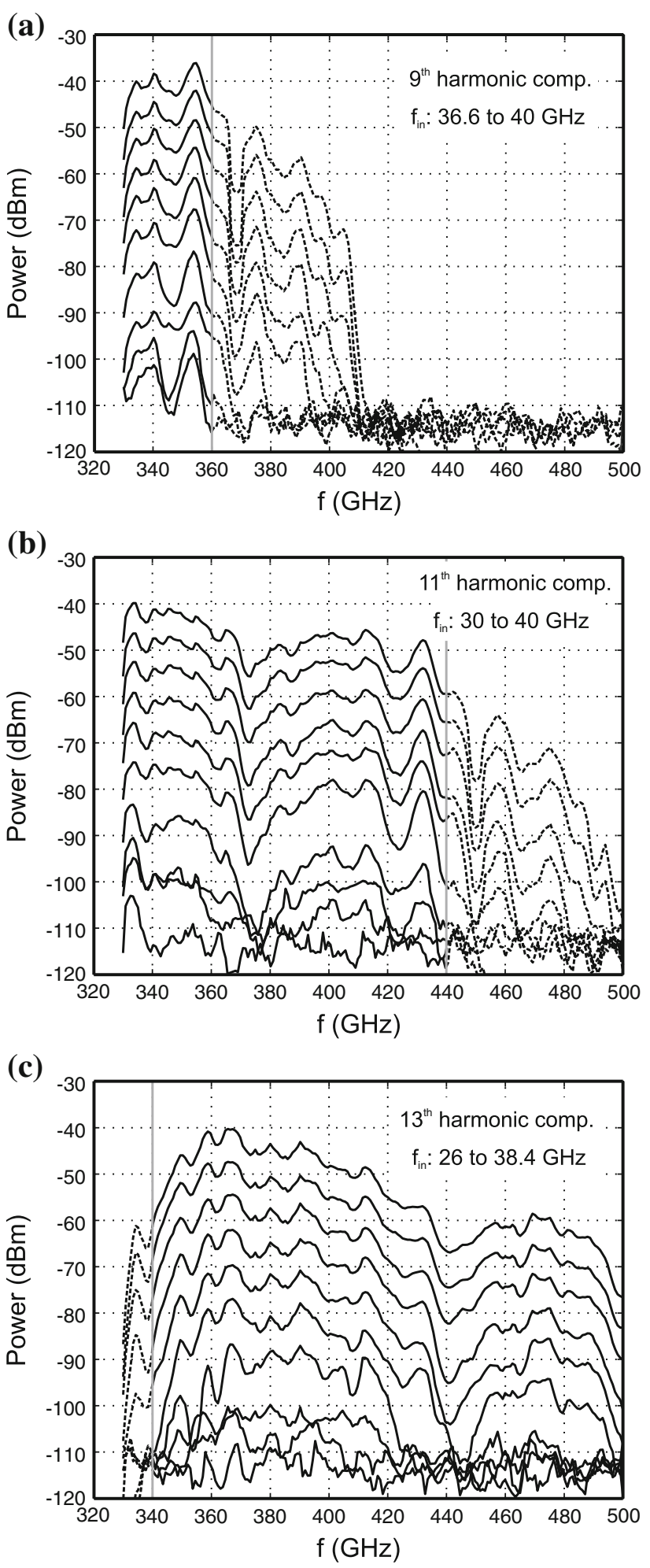

Fig. 5 Measured output power of odd-order harmonic components. In all cases, the input signal power varies in $2 \mathrm{~dB}$ steps, with a $-6 \mathrm{dBm}$ maximum. a $N=9$. Input frequency from 36.6 to $40 \mathrm{GHz}$. b $N=11$. Input frequency from 30 to $40 \mathrm{GHz}$. c $N=13$. Input frequency from 26 to $38.4 \mathrm{GHz}$ 



Fig. 6 Measured output power of odd-order harmonic components. In all cases, the input signal power varies in $2 \mathrm{~dB}$ steps, with a $-6 \mathrm{dBm}$ maximum. a $N=15$. Input frequency from 26 to $33.4 \mathrm{GHz}$. b $N=17$. Input frequency from 26 to $29.4 \mathrm{GHz}$

the elements of the measurement setup represented in Fig. 4. In any case, the frequency multiplication phenomenon is observed along the entire considered frequency bands. On the other hand, the output power reduction that is observed for frequency values greater than $450 \mathrm{GHz}$ is due to the fact that $S_{12}$ parameter represented in Fig. 3b worsens from this frequency point, which means that a portion of the signal generated in the $450-500 \mathrm{GHz}$ frequency range is not coupled to the output waveguide. A similar effect appears in the low-frequency region represented in Fig. 5c and Fig. 6. In this case, since the input port is not well impedance matched from 26 to about $27.5 \mathrm{GHz}$ (see $S_{11}$ parameter in Fig. 3a), the output power of the 13th harmonic component under $13 \times 27.5=$ $357.5 \mathrm{GHz}$, the 15 th harmonic component under $15 \times$ $27.5=412.5 \mathrm{GHz}$ and the $17 \mathrm{th}$ harmonic component

Fig. 7 Measured output power of even-order harmonic components. In all cases, the input signal power varies in $2 \mathrm{~dB}$ steps, with a $-6 \mathrm{dBm}$ maximum. a $N=10$. Input frequency from 33 to $40 \mathrm{GHz}$. b $N=12$. Input frequency from 27.5 to $40 \mathrm{GHz}$

under $17 \times 27.5=467.5 \mathrm{GHz}$ is lower than expected. In any case, the bandwidth in which the frequency multiplication effect is obtained is only limited by the additional necessary circuitry and the measurement system, but not by the graphene sheet itself.

\subsection{Even-order harmonic components}

The variation of the output power of the even-order harmonic components, from $N=10$ to $N=16$, has been represented in Figs. 7 and 8. As in the previous case, the power of the input signal varies in $2 \mathrm{~dB}$ steps, with a maximum value of $-6 \mathrm{dBm}$. In this case, for the same value of the input power, the measured output power is considerably lower than the obtained when evaluating the odd-order harmonic components. This fact is in accordance 

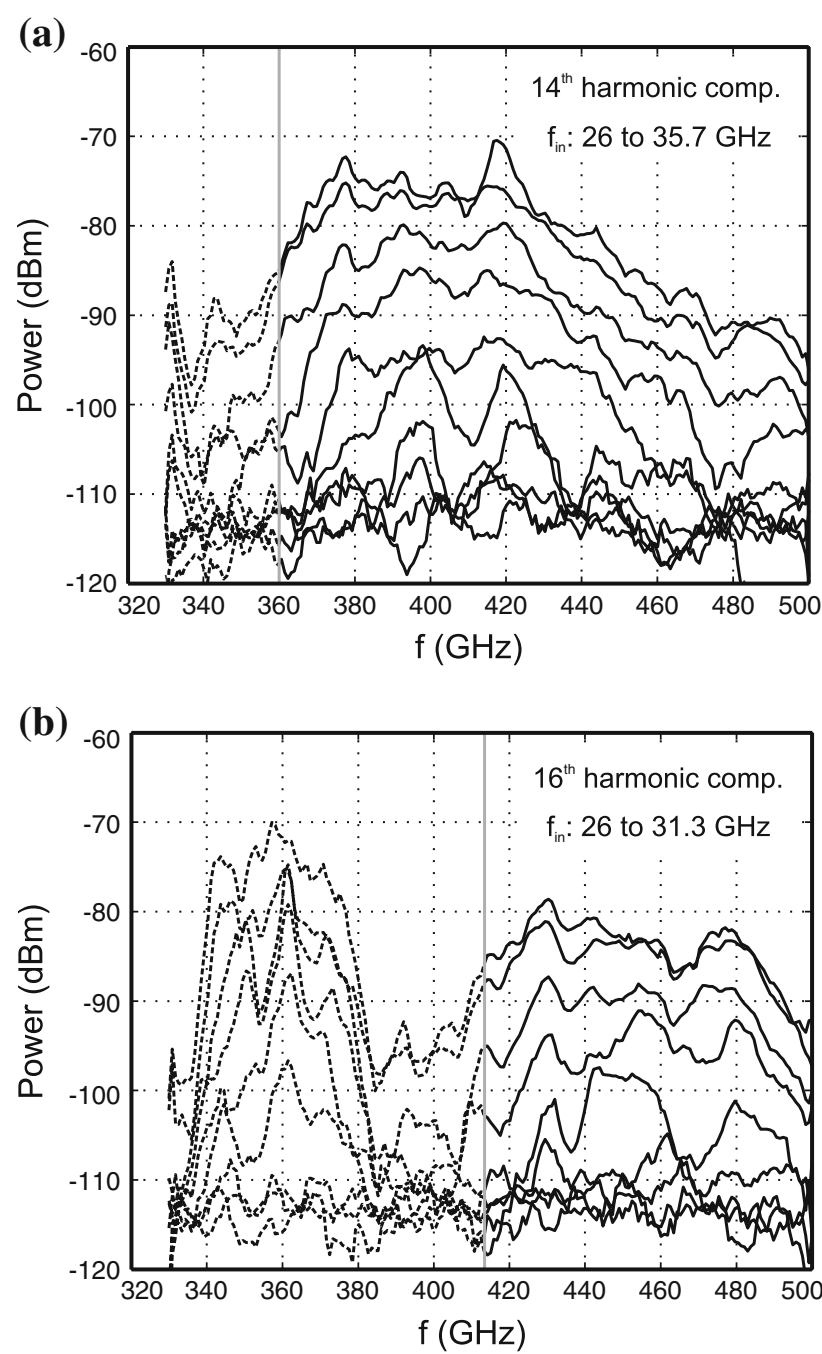

Fig. 8 Measured output power of even-order harmonic components. In all cases, the input signal power varies in $2 \mathrm{~dB}$ steps, with a $-6 \mathrm{dBm}$ maximum. a $N=14$. Input frequency from 26 to $35.7 \mathrm{GHz}$. b $N=16$. Input frequency from 26 to $31.3 \mathrm{GHz}$

with the graphene behavior theory [3], which predicts the excitation of odd harmonic components when the graphene is excited with an incident electromagnetic wave. Although the appearance of even-order harmonic components of the exciting wave is not considered, it is necessary to take into account the differences with respect to this work. While in [3] an ideal infinite two-dimensional isolated graphene layer is analytically studied, the experimental results presented in this work have been obtained using a finite fewlayer graphene sheet which interacts with the polyimide substrate.

\subsection{Output voltage versus the harmonic order}

The theoretical analysis reported in [3] shows that the amplitude of the odd-order harmonic components generated

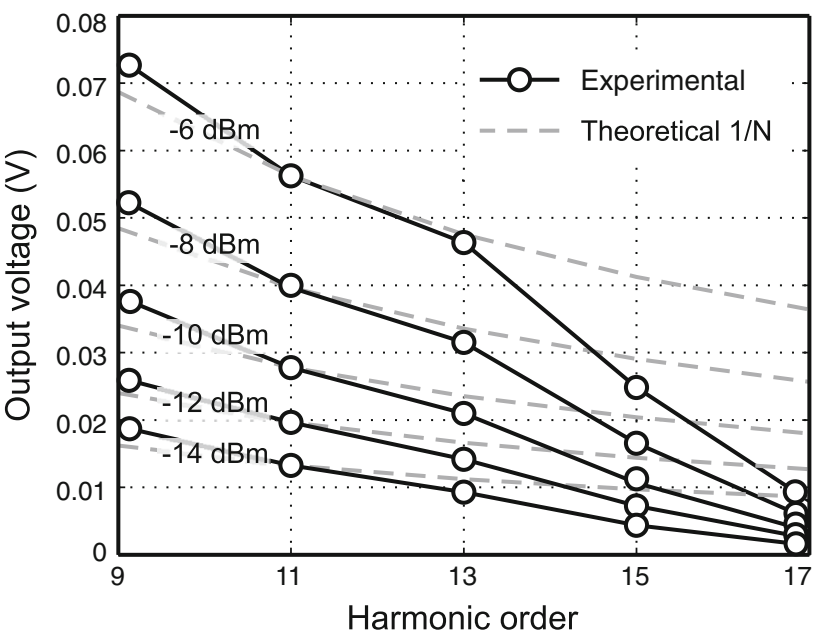

Fig. 9 Variation of the output power with the harmonic order for fixed values of the input signal power. Only odd-order harmonic components are considered

when the ideal graphene layer is excited with an electromagnetic wave decreases as the inverse of the harmonic order. Considering the measured output power of the oddorder harmonic components represented in Figs. 5 and 6 and assuming a $50 \Omega$ reference impedance, the voltage amplitude associated with each trace has been computed. Next, its mean value along the corresponding nominal frequency range, for a fixed value of the input power, has been calculated. The mean value of the amplitude is considered instead of the value obtained for a particular frequency point because there is no one common to all the odd-order harmonic components. The result has been represented in Fig. 9, together with the theoretical 1/ $N$ variation law (dashed line). As shown, in the case of 9 , 11 and 13 harmonic orders, the experimental results agree with the theoretical prediction. However, the amplitude of the 15th and the 17th harmonic components decreases faster than $1 / N$. This fact can be explained taking into account that the frequency of such harmonic components is located in the frequency region in which the generated signal is not fully coupled to the output waveguide (see Fig. 3b).

The presented experimental results show that few-layer graphene exhibits a nonlinear response when it is excited with an electromagnetic wave. The observed generation of high-odd-order harmonic components of the incident wave and the evolution of their amplitude with the harmonic order are in accordance with the theoretical studies referred to an isolated ideal two-dimensional graphene layer. In addition, the developed assembly represents a proof of concept which demonstrates that few-layer graphene can be used, in a simple configuration, as $\mathrm{THz}$ signal source based on frequency multiplication. 


\section{Conclusions}

In this work, the nonlinear electromagnetic response of a few-layer graphene sheet obtained through mechanical exfoliation from a highly ordered pyrolitic graphite block has been experimentally analyzed. The graphene sheet is part of a stripline circuit which is embedded in a metallic waveguide structure used to guide the exciting input and the output signals. The few-layer graphene sheet is used as frequency multiplier to generate high-order harmonic components of the $26-40 \mathrm{GHz}$ pump signal, from order 9 to 17 . The achieved results show that the output power of the even-order harmonic components is considerably lower than the reached in the case of odd-order harmonic components. On the other hand, for a fixed value of the input power, the amplitude of the odd-order harmonic components decreases as the inverse of the harmonic order. In addition, the bandwidth in which the described behavior is obtained is only limited by the characteristics of the circuitry, but not by the graphene sheet. Therefore, although the experimental results have been obtained from a nonisolated finite few-layer graphene, they are in accordance with the previously reported theoretical studies. Finally, the described assembly represents a proof of concept which demonstrates that graphene can be used by itself as signal generator in the $\mathrm{THz}$ band.

Acknowledgments This work has been partially supported by the VII Framework Programme of the EUROPEAN UNION under Project ICT-2011.8.2 600849, by the MINISTERIO DE CIENCIA E INNOVACIÓN of Spain and FEDER under the Projects IPT-20110951-390000, TEC2011-24492, TEC2008-01638 and CONSOLIDER INGENIO CSD2008-00068, and the Grant AP2012-2020, by the GOBIERNO DEL PRINCIPADO DE ASTURIAS (PCTI/FEDERFSE) under the Projects EQUIP08-06, FC09-COF09-12 y EQUIP1031, and the Grant BP13034, and by the CÁTEDRA TELEFÓNICAUNIVERSIDAD DE OVIEDO.

\section{References}

1. K.S. Novoselov, A.K. Geim, S.V. Morozov, D. Jiang, Y. Zhang, S.V. Dubonos, I.V. Grigorieva, A.A. Firsov, Science 306, 666 (2004)

2. M.M. Glazov, S.D. Ganichev, Phys. Rep. 535, 101 (2014)

3. S.A. Mikhailov, EPL 79, 27002-p1 (2007)

4. M. Dragoman, D. Neculoiu, G. Deligeorgis, G. Konstantinidis, D. Dragoman, A. Cismaru, A.A. Muller, R. Plana, Appl. Phys. Lett. 97, 093101 (2010)

5. J.J. Dean, H.M. van Driel, Appl. Phys. Lett. 95, 261910 (2009)

6. J.J. Dean, H.M. van Driel, Phys. Rev. B 82, 125411 (2010)

7. A.Y. Bykov, T.V. Murzina, M.G. Rybin, E.D. Obraztsova, Phys. Rev. B 85, 121413 (2012)

8. N. Kumar, J. Kumar, C. Gerstenkorn, R. Wang, H.Y. Chiu, A.L. Smirl, H. Zhao, Phys. Rev. B 87, 121406 (2013)

9. S.Y. Hong, J.I. Dadap, N. Petrone, P.C. Yeh, J. Hone, R.M. Osgood Jr., Phys. Rev. X 3, 021014 (2013)

10. R. Camblor, S. Ver Hoeye, G. Hotopan, C. Vazquez, M. Fernandez, F. Las Heras, P. Alvarez, R. Menendez, J. Electromagn. Waves Appl. 25, 1921 (2011)

11. G. Hotopan, S. Ver Hoeye, C. Vazquez, R. Camblor, M. Fernandez, F. Las Heras, P. Alvarez, R. Menendez. Prog. Electromagn. Res. 118, 57 (2011)

12. N.L. Rangel, A. Gimenez, A. Sinitskii, J.M. Seminario, J. Phys. Chem. C 115, 12128 (2011)

13. G. Hotopan, S. Ver Hoeye, C. Vazquez, A. Hadarig, R. Camblor, M. Fernandez. Prog. Electromagn. Res. 140, 781 (2013)

14. K.N. Parrish, D. Akinwande, Appl. Phys. Lett. 99, 223512 (2011)

15. H. Wang, D. Nezih, J. Kong, T. Palacios, IEEE Electron Device Lett. 30(5), 547 (2009)

16. Z. Wang, Z. Zhang, H. Xu, L. Ding, S. Wang, L.-M. Peng, Appl. Phys. Lett. 96, 173104 (2010)

17. L. Liao, Y.-C. Lin, M. Bao, R. Cheng, J. Bai, Y. Liu, Y. Qu, K.L. Wang, Y. Huang, X. Duan, Nature 467, 305 (2010)

18. D.M. Pozar, Microwave Engineering, 2nd edn. (Wiley, New York, 1998) 